\title{
HEMATOPOIETIC STEM CELL SEPARATION FOR EXPERIMENTAL PURPOSES - METHODIC LIMITATIONS
}

\author{
Pavla Luzna $^{\text {a**, Dana Kylarova }}{ }^{\mathrm{a}}$, Martin Novak ${ }^{\mathrm{b}}$, Vaclav Lichnovsky ${ }^{\mathrm{a}}$ \\ a Department of Histology and Embryology, Faculty of Medicine and Dentistry, Palacky University, 77515 Olomouc, Czech \\ Republic \\ ${ }^{b}$ Department of Hemato - Oncology, University Hospital, 77520 Olomouc, Czech Republic \\ e-mail:pavla83@hotmail.com
}

Received: October 21, 2008; Accepted: February 19, 2009

Key words: Stem cells/CD117/Immunomagnetic separation/Immunofluorescence

Background: Protooncogene CD117 is a cytokine receptor important for hematopoietic element development. Currently, we are not able to routinely separate a sufficient quantity of bone marrow CD $117^{+}$cells for experimental purposes.

Aim: The aim of this study was to establish an immunomagnetic separation method for $\mathrm{CD} 117^{+}$hematopoietic stem cell isolation and to estimate the expression of chosen $\mathrm{BCl}-2$ family protein members in these elements.

Material and Methods: 120 samples of human and murine bone marrow were acquired using the magnetic separation system. The cells were stained for $\mathrm{CD} 117, \mathrm{BCl}-2, \mathrm{BAX}$, and $\mathrm{CD} 33$ by an indirect fluorescent immunocytochemistry.

Results: The flow cytometry analysis showed only $2,6 \% \mathrm{CD} 117^{+}$cells from human as well as mouse bone marrow which is insufficient for further experiments. Cytospin was not good for morphologic characterization and immunophenotyping due to the fragility and destruction of the studied cells. Therefore, cell suspension staining was selected and by this method we found CD117 positivity in $70 \%$ of the mononuclerar (CD33 positive) elements in the case of chronic myeloid leukaemia. Labelling of the $\mathrm{BCl}-2$ family in this case showed antiapoptotic $\mathrm{BCl}-2$ expression in $80 \%$, proapoptotic BAX expression in approximately $5 \%$.

Conclusion: Our results show that CD117 immunomagnetic separation from bone marrow material is not acceptable for experimental purposes. They demonstrate that the only practical useful for the bone marrow cell examination (morphology and immunophenotype) is cell suspension staining which uncovers the distribution of both cytoplasmic proteins and surface antigens of immature blood elements.

\section{INTRODUCTION}

CD117 (KIT/C-kit receptor) is a cytokine receptor expressed on the surface of hematopoietic stem cells ${ }^{1,2}$. Signalling through CD117 has an influence on cell survival, proliferation, and differentiation and plays an important role in hematopoietic element development. CD117 is a protooncogene and its overexpression or mutation can lead to cancer ${ }^{1,2}$. Percentage estimation of CD $117^{+}$ elements in bone marrow samples is already routinely made using flow cytometry as a step in the patient's immunophenotype profiling. However, we are not able to separate a sufficient quantity of $\mathrm{CD} 117^{+}$cells during routine processes for further experimental uses. One of the applications $\mathrm{CD} 117^{+}$cells is study of the expression of apoptosis regulating proteins such as the $\mathrm{BCl}-2$ protein family which is important for normal development of blood cells ${ }^{3}$. One generally accepted method for isolating cells is immunomagnetic separation ${ }^{4}$.

\section{AIM}

To establish an immunomagnetic separation method for $\mathrm{CD} 117^{+}$hematopoietic stem cells and to estimate the expression of $\mathrm{BCl}-2$ family proteins in human bone marrow samples.

\section{MATERIAL AND METHODS}

The bone marrow samples were acquired from the Hemato-Oncology Clinic, Faculty of Medicine and Dentistry, Palacky University Olomouc and University Hospital, Olomouc. The biopsy of the bone marrow was taken as part of routine diagnosis and was approved by the local Ethics Committee.

We took 120 samples of 1-2 $\mathrm{ml}$ of bone marrow harvested in $10 \mathrm{ml}$ of RPMI medium (Sigma Aldrich, St. Louis, MO, USA), enriched by $500 \mu 1$ 7,5\% $\mathrm{NaHCO}_{3}$ (Sevapharma, Prague, CZ) and $200 \mu \mathrm{l}$ heparin (500 IU/ $\mathrm{ml}$ ), (Zentiva, Prague, CZ). Briefly, the mononuclear cells were acquired by the Histopaque ${ }^{\circledR}$ gradient centrifugation (Sigma-Aldrich, Prague, CZ). Murine bone marrow was acquired from the C57/BL6 mouse. Healthy mice were sacrificed, the total bone marrow was extracted and a mononuclear suspension was prepared as described above.

We used the magnetic separation system from the StemCell Technologies ${ }^{\mathrm{TM}}$ (Vancouver, Canada) described 


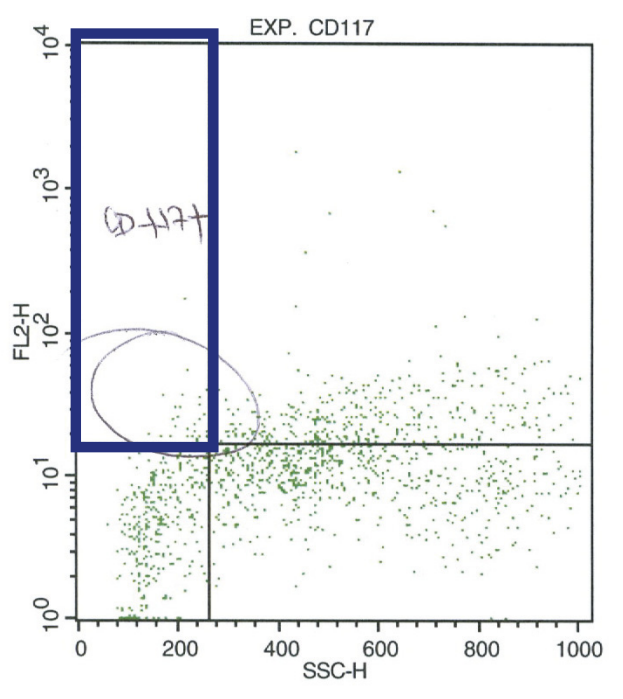

Quad Location: 261, 17

$$
\begin{array}{rrr}
\text { Quad } & \text { Events } & \% \text { Gated } \\
\hline \text { UL } & 106 & 2.57
\end{array}
$$

Fig. 1. Flow cytometer analysis of separated CD117 cells. The blue rectangle shows the number of CD117 separated cells (2.6\%). SSC - Side scatter (gradient of the cellular composition). FL2H - fluorescence channel 2- high, channel for labelled cells measuring. UL - upper left quadrant.

by Wognum et al. ${ }^{4}$, Chen et al. ${ }^{5}$. Shortly, the system consists of a magnet generating a strong magnetic field and a tube containing the cell suspension. The cell suspension is incubated with the antibody aimed at the antigen of interest. The antibody is then crossed with the magnetic nanoparticles and the labelled cell suspension is then placed into the magnet. After 5 minutes the supernatant is poured off and the positively labelled cells remain in the tube. The cells are then washed several times. The recommended starting quantity for the separation process is between $10^{8}$ and $10^{9}$ cells.

The amount of separated cells was assessed by flow cytometric analysis (BD FACSCalibur ${ }^{\mathrm{TM}}$ system, BD Biosciences, Franklin Lakes, NJ, USA).

Mononuclear cells were obtained from newly diagnosed chronic myeloid leukemia (CML) patients and CD33 staining (Santa Cruz Biotechnology, Santa Cruz, CA, USA, dilution 1:50) was used as marker of myeloid cell population ${ }^{6}$.

Cytospin slides were prepared as follows: (cytocentrifugation, 600 RPM), fixed in methanol : acetone (1:1) for 5 minutes and subsequently CD117 (Santa Cruz Biotechnology, Santa Cruz, CA, USA, dilution 1:50) was stained by indirect double-step immunocytochemistry with FITC conjugated secondary antibody (Jackson Immuno Research, West Grove, PA, USA, dilution 1:50). The nuclei were counterstained by DAPI (Millipore, Billerica, MA, USA). Also CD33 (Santa Cruz Biotechnology, Santa Cruz, CA, USA, dilution 1:50) an-
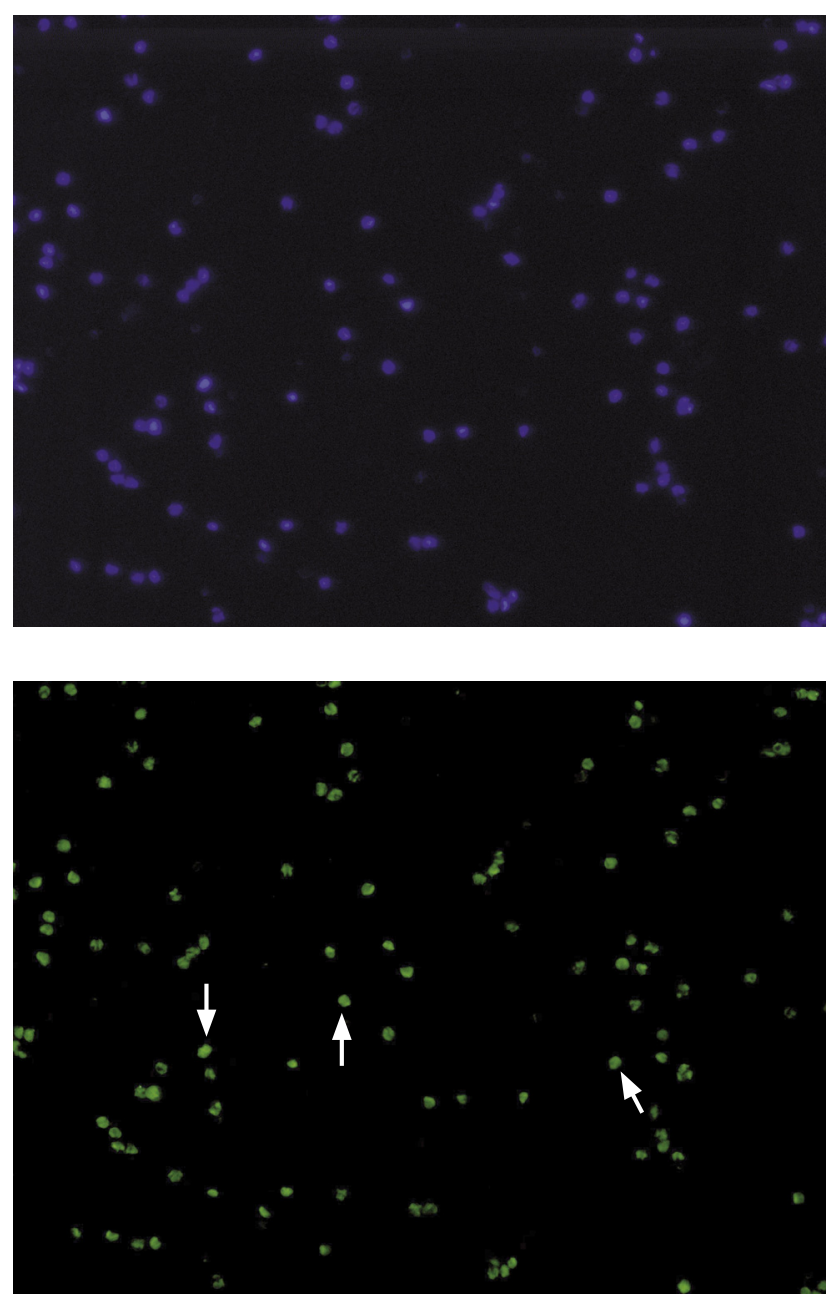

Fig. 2. CD117 indirect double-step fluorescent cell suspension labelling.

a. DAPI staining of the nuclei

b. CD117 positive cells (FITC dye, examples of positivity showed by arrow) all magn.x 20

tigen was labelled, the secondary antibody was used as described above. Selected BCL-2 family proteins [BCl-2 (Biogenex, San Ramon, CA, USA, dilution 1:100), BAX (Santa Cruz Biotechnology, Santa Cruz, CA, USA, dilution 1:50)] were stained by indirect three step immunofluorescence [secondary antibody biotinylated anti-mouse $\operatorname{IgG}(\mathrm{H}+\mathrm{L})$ (Jackson Immuno Research, West Grove, PA, USA), Texas $\operatorname{Red}^{\circledR}$ dye conjugated Streptavidin (Vector Laboratories, Burlingame, CA, USA)].

Cell suspension staining: the cell suspension was spread directly onto a microscopic slide before fixation and immunostaining (see above cytospin staining).

\section{RESULTS AND DISCUSSION}

The flow cytometry analysis showed that we separated only $2.6 \% \mathrm{CD} 117^{+}$cells (Fig. 1) from human bone marrow which is insufficient for further experiments. For this 

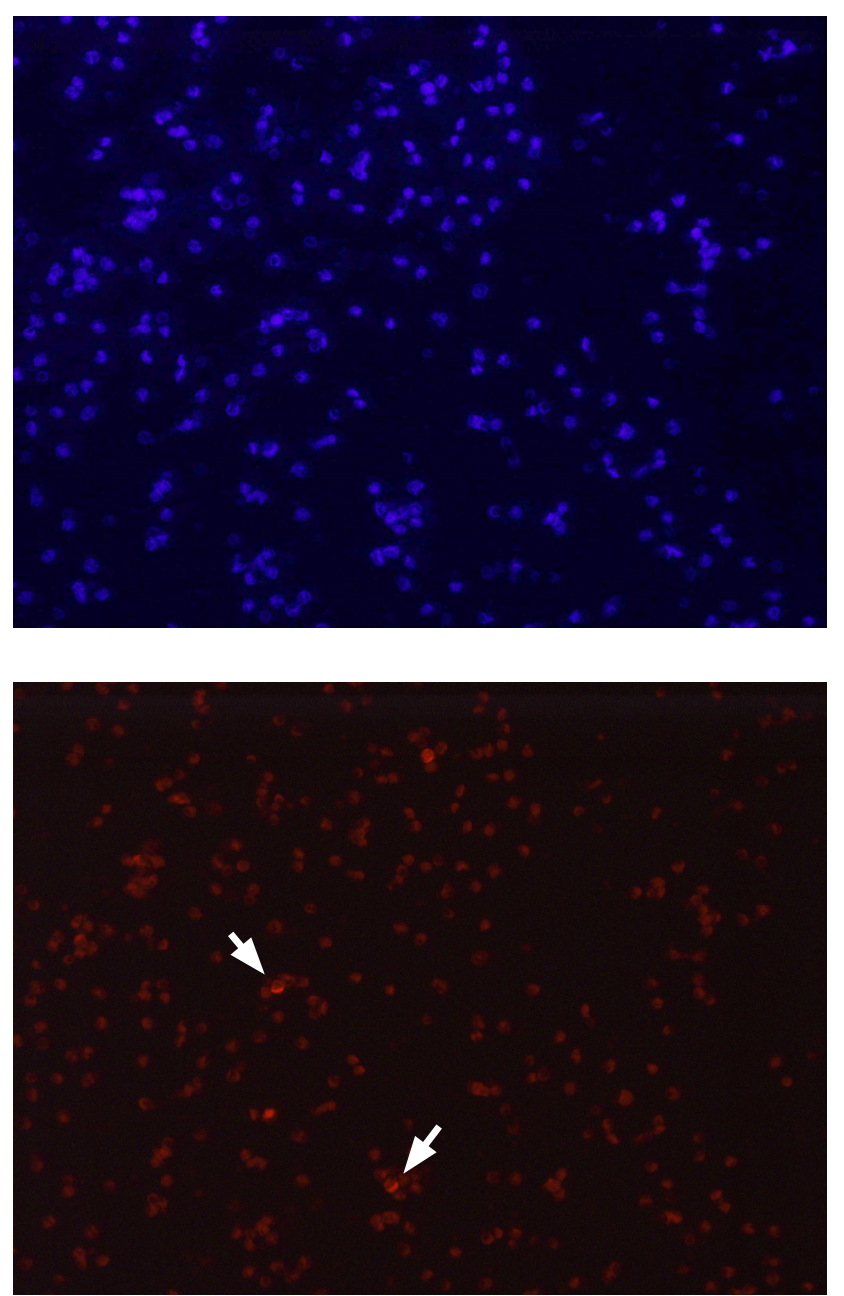

Fig. 3. BAX indirect three-step fluorescent cell suspension labelling.

a. DAPI staining of the nuclei

b. BAX positive cells (TexasRed ${ }^{\circledR}$ dye, examples of positivity showed by arrow) all magn.x20

reason, we decided to establish immunomagnetic cell separation from murine material but the amount of separated cells was also insufficient ( $1 \%$ ). We then did the cytospin for the detection of cells of interest. Although the cytospin was done carefully, the fragile blood elements were destroyed. Therefore, the most simple methodical approach - cell suspension staining was selected. Mononuclear cells from the newly diagnosed CML patient were chosen for this staining. As we expected, due to the large number of immature cells, CD117 positivity was found in $70 \%$ of the mononuclear elements (Fig. 2). CD33 positivity was counted in $60 \%$ of mononuclear cells. Further, the labelling of the Bcl-family proteins showed antiapoptotic BCl-2 expression in $80 \%$, in contrast to proapoptotic BAX which had weak positivity (approximately $5 \%$ ) (Fig. 3). Therefore, we assumed that the large number of CD $117^{+}$ cells was a result of the leukaemic origin of the cells.

\section{CONCLUSIONS}

Our results show, that CD117 immunomagnetic separation from residual bone marrow material is not acceptable for experimental purposes. The initial number of cells does not fulfill the conditions for the separation process. Our results demonstrated that the only practical approach for bone marrow cell examination (morphology and immunophenotype) is cell suspension staining which reveals both the distribution of cytoplasmic proteins and surface antigens of the immature blood elements.

\section{REFERENCES}

1. Miettinen M, Lasota J. KIT (CD117): a review on expression in normal and neoplastic tissues, and mutations and their clinicopathologic correlation. Appl Immunohistochem Mol Morphol 2005; 13(3):205-20.

2. Rönnstrand L. Signal transduction via the stem cell factor receptor/ c-Kit. Cell Mol Life Sci. 2004; 61(19-20):2535-48.

3. Oguro H, Iwama A. Life and death in hematopoietic stem cells. Current Opinion in Immunology 2007; 19:503-509.

4. Wognum AW, Eaves AC, Thomas TE. Identification and isolation of hematopoietic stem cells. Arch Med Res. 2003; 34(6):461-75.

5. Chen W, Antonenko S, Sederstrom JM, Liang X, Chan AS, Kanzler $\mathrm{H}$ et al. Thrombopoietin cooperates with FLT3-ligand in the generation of plasmacytoid dendritic cell precursors from human hematopoietic progenitors. Blood 2004; 103(7): 2547-53.

6. Freeman SD, Kelm S, Barber EK, Crocker PR. Characterization of CD33 as a new member of the sialoadhesin family of cellular interaction molecules. Blood 1995; 85(8): 2005-12. 
